\title{
2 A common polymorphism that protects from cardiovascular disease increases 3 fibronectin processing and secretion
}

5 Sébastien Soubeyrand, $\mathrm{PhD}^{\mathrm{a} *}$, Paulina Lau MSc ${ }^{\mathrm{a}}$, Majid Nikpay, PhD ${ }^{\mathrm{a}}$, Anh-Thu Dang MSc ${ }^{\mathrm{a}}$, 6 and Ruth McPherson, $\mathrm{MD}, \mathrm{PhD}^{\mathrm{a}, \mathrm{b} *}$.

$8{ }^{a}$ Atherogenomics Laboratory, University of Ottawa Heart Institute, Ottawa, Canada; ${ }^{b}$

9 Department of Medicine, Ruddy Canadian Cardiovascular Genetics Centre, University of Ottawa 10 Heart Institute, Ottawa, Canada.

* To whom correspondence may be addressed:

14 ssoubeyrand@ottawaheart.ca

15 rmcpherson@ottawaheart.ca

16

17

Author Contributions: S.S. and P.L designed, performed and analyzed experiments; A.-T. D performed experiments; M.N. completed bioinformatic analyses; R.M. and S.S. wrote the manuscript.

Competing Interest Statement: The authors declare no competing interests.

Keywords: Fibronectin 1, coronary artery disease, Single nucleotide polymorphism, signal peptide, glycosylation. 
31 Background: Fibronectin $(F N 1)$ is an essential regulator of homodynamic processes and tissue

32 remodeling which has been proposed to contribute to atherosclerosis. Moreover, recent large scale genome wide association studies have linked common genetic variants within the $F N 1$ gene to coronary artery disease $(\mathrm{CAD})$ risk.

Methods: Public databases were analyzed by two-Sample Mendelian Randomization. Expression constructs encoding short FN1 reporter constructs and full-length plasma $F N 1$,

37 differing in the polymorphism, were designed and introduced in various cell models. Secreted

38 and cellular levels were then analyzed and quantified by SDS-PAGE and fluorescence approaches. Mass spectrometry and glycosylation analyses were performed to probe possible post-transcriptional differences.

Results: Higher FN1 protein levels in plasma associates with a reduced risk of cardiovascular disease. Moreover, common CAD risk SNPs in the FN1 locus associate with circulating levels of FN1. This region is shown to encompass a L15Q polymorphism within the FN1 signal peptide. The presence of the minor allele that predisposes to $\mathrm{CAD}$, corresponding to the Q15 variant, alters glycosylation and reduces FN1 secretion in a direction consistent with the bioinformatic analyses.

47 Conclusion: In addition to providing novel functional evidence implicating FN1 as a protective

48 force in cardiovascular disease, these findings demonstrate that a common variant within a secretion signal peptide regulates protein function. 
bioRxiv preprint doi: https://doi.org/10.1101/2021.03.09.434522; this version posted April 5, 2021. The copyright holder for this preprint (which was not certified by peer review) is the author/funder, who has granted bioRxiv a license to display the preprint in perpetuity. It is made available under aCC-BY 4.0 International license.

53

54 


\section{Introduction}

57 Genome-wide Association Studies (GWAS) have identified hundreds of common single nucleotide polymorphisms (SNPs) that associate with cardiovascular disease (CAD) risk ${ }^{1-3}$.

59 Although GWAS signals are enriched for expression quantitative trait loci (eQTLs) indicating measurable impacts on transcription, the identification of causal genes is challenging since 1) the vast majority of common trait related SNPs do not overlap protein coding genes and 2) are

62 eQTLs for multiple genes ${ }^{4,5}$. Validation of statistical associations by experimental approaches is an essential first step in the development of novel therapeutic interventions. As the majority of

64 GWAS identified variants are unlikely to be causal for several reasons, the very identification of causal SNPs among the list of GWAS identified variants is itself a complex process ${ }^{6}$. Indeed, predictions place at least $80 \%$ of GWAS identified SNPs within a substantially wide $34 \mathrm{kbp}$ window of causal variants in Europeans ${ }^{7}$. Clearly, mechanistic insights are limited at this level of resolution, especially since trans (long-distance) acting variants are prevalent and may account for significant heritability ${ }^{8}$. In order to pinpoint causal SNPs ("finemapping") and identify functionally important gene targets, various approaches have been used that leverage expression data, epigenetic information, etc. ${ }^{9}$. This approach has yielded surprising findings

72 including variants located within and outside genes that regulate distal genes, as well as evidence 73 of pervasive transcription independent mechanisms ${ }^{10-12}$.

The Fibronectin 1 gene (FN1) encodes a group of protein isoforms that differ in sequence 75 and localization: plasma $(\mathrm{pFN})$ and cellular $(\mathrm{cFN})^{13}$. Both forms are synthesized as precursors that are processed during ER/Golgi trafficking and either enrich the local matrix environment

$77(\mathrm{cFN})$ or secreted into the circulation $(\mathrm{pFN}){ }^{14}$. The cellular forms exist as multiple variants that act as key structural components and regulators of the extracellular matrix (ECM), where they 
79 are deposited as insoluble fibers involved in cell adhesion. The second major form of FN1, pFN,

80 is secreted by the liver into the circulation where it is abundant. Mice deficient in pFN display

81 largely normal hemostasis and wound-healing, consistent with a predominant role of cFN rather

82 than $\mathrm{pFN}$ in these processes ${ }^{15}$. Interestingly, $\mathrm{pFN}$ deficient mice display increased neuronal

83 apoptosis and larger infarct areas following focal brain ischemia, suggesting that pFN plays a

84 protective role, possibly by activating anti-apoptotic mechanisms via integrin signaling ${ }^{15}$. While

$85 \mathrm{pFN}$ is not essential to vascular integrity, $\mathrm{pFN}$ has been shown to penetrate the vessel wall and to

86 constitute a significant portion of arterial $\mathrm{FN}$ where it may participate in normal tissue

87 remodeling, angiogenesis and neoplasia ${ }^{16-19}$.

Here, we explore and clarify the mechanisms linking common GWAS identified variants that map to the FNlgene to CAD risk. Using bioinformatic and molecular approaches we provide evidence that differential post-transcriptional regulation underlies the FN1-CAD association. More specifically a polymorphism within the signal peptide of FN1 is found to regulate the ability of FN1 to be secreted. These findings provide a unique portrait of a common

93 coding variant linked to $\mathrm{CAD}$ that has functional consequences at the protein level without

94 affecting its mature amino acid sequence. 


\section{Results}

\section{rs1250259 links FN1 protein expression to coronary artery disease}

The CAD-linked haplotype harbors several tightly linked SNPs that correlate with the disease

116 SNP contributions, circulating FN1 (probe) and CAD were inversely correlated, with higher

117 circulating FN1 linked to lower CAD risk (Table 1). Moreover, the 2-sample design supports a

118 causal contribution of FN1, although reverse causation (CAD affecting FN1 levels) cannot be 
122 Table 1. Mendelian randomization reveals an impact of FN1 on CAD. Probes (protein

123 concentration) and corresponding CAD values are from Suhre et al ${ }^{24}$. Bxy, regression

124 coefficient of $x$ and $y$; se, Standard Error; $p$, pvalue of the beta; nsnp: number of snps used in

125 model; Z, Z-score of the correlation. All values are rounded to 2 significant figures.

126

\begin{tabular}{|c|c|c|c|c|c|c|}
\hline Probe & Outcome & bxy & se & $p$ & nsnp & Z \\
\hline 3434-34_1 & CAD & -0.059 & 0.0094 & $3.70 \mathrm{E}-10$ & 5 & -6.3 \\
\hline 4131-72_2 & CAD & -0.061 & 0.0094 & $1.00 \mathrm{E}-10$ & 4 & -6.5 \\
\hline
\end{tabular}

127

128 
Identification of a missense mutation within FN1 linked to CAD that is predicted to affect secretion

Although the above analysis focused on FN1 protein expression, the contribution to FN1 mRNA expression remained to be tested. Genotype-Tissue Expression (GTEx) data indicate that the CAD linked haplotype region (including rs1250229 and rs1250259) was not associated with statistically significant changes in FN1 expression in any of the available tissues (data not shown). This suggests that the haplotype may affect (harder to detect) distal genes, tissues not

137 part of the GTEx panel or a combination thereof. Alternatively, the region may affect FN1 posttranscriptionally. Translation of rs 1250259 is predicted to yield protein variants harboring either a Gln (rs1250259-T) or Leu (rs1250259-A) at position 15. Of note, the SNP haplotype is defined synthesized as a precursor that undergoes removal of a $\sim 30$ amino acid region containing a hydrophobic signal peptide (which includes residue 15) and a hydrophilic short pro-sequence ${ }^{14}$.

\section{The rs1250259 affects secretion of a FN1 fusion construct in transformed and primary cell} models

To examine the impact of this substitution on FN1 secretion, a model fusion protein consisting of

147 amino acids 1-182 of FN1 fused to a GFP-HA tag moiety was generated (Figure 3A). This

148 moiety is conserved in all FN1 forms and addresses technical limitations linked to the large size

149 of FN1. The FN1 region chosen corresponds to the N-terminal heparin binding domain, which

150 forms a well-defined region by crystallography and NMR and is shared by both secreted and 151 cellular forms. Expression was first tested in HEK293T, a readily transfectable and widely 
available cell line. Following SDS-PAGE of cell lysates, a shift was observed: the Q15 form migrates slightly slower than the L15 variant (Figure 3B). Both fusion variants were present at comparable levels in HEK293T lysates after correcting for transfection efficiency (Figure 3 C).

Presence of the secreted protein in the media was tested next (Figure 4). In HEK293T and HeLa cells, transfection resulted in the secretion of FN1-GFP in the media, with the L15 exhibiting greater propensity to be secreted, defined as the signal in the culture media relative to the cellular signal. To examine the impact of this polymorphism on secretion by the liver, which is the major physiological source of $\mathrm{pFN} 1, \mathrm{HuH}-7$ hepatoma cells, a widely used model of hepatocyte function, were transfected. Although the difference was smaller than observed in the epithelial models above, consistently L15 FN1-GFP was more readily secreted by HuH-7 cells. Finally, FN1-GFP was transduced into several primary cell models with relevance to CAD, i.e., adventitial fibroblasts, endothelial cells, and coronary smooth muscle cells. In all models, the Leu form was on average better secreted than the Gln form, although the difference reached statistical significance only in a lot of coronary smooth muscle cells.

\section{Secreted forms of Q15 qualitatively differ in primary cells}

Examination of the variants by SDS-PAGE revealed some unexpected findings. Delivery of FN1-GFP demonstrated isoform-specific differences in the secreted forms, in a cell-type different manner (Figure 5). In some cells (fibroblasts, smooth muscle models as well as HeLa cells), transduction of the Q15 form led to enrichment relative to the L15 form of a slower migrating band on SDS-PAGE. By contrast, FN1-GFP from endothelial cells and HEK293T resembled HuH-7 cells in that both secreted forms exhibited qualitatively more similar profiles. 
174 Thus, in some cell types, the L15Q polymorphism appears to dictate both the quality and 175 quantity of FN1-GFP secreted.

176

\section{Differences in O-glycosylation account for the difference in migration}

We hypothesized that this 3-5 $\mathrm{kDa}$ difference was due to variable levels of posttranslational modification, possibly glycosylation and/or retention of pro-peptides of different lengths. As full-length pFN1 is modified post-transcriptionaly by $\mathrm{O}$ and N-linked glycosylation, events commonly associated with secretion, glycosylation was examined first. Both variants secreted from dermal fibroblasts were subjected to deglycosylation reactions in vitro using a cocktail of enzymes targeting a wide range of glycosylation chains. The incubation resulted in the disappearance of the slower migrating form (Figure 6). Interestingly, a longer exposure of the L15 form also shows the presence of a slower migrating band that is also sensitive to glycosylation treatment. Thus, both forms are glycosylated, albeit to different extent.

The type of glycosylation involved was examined by treating cells with tunicamycin, which blocks N-glycosylation thereby interfering with protein transit through the Golgi apparatus and secretion. Inclusion of tunicamycin severely reduced the amount, and altered the migration, of full-length endogenous FN1 recovered from the media but its impact on FN1-GFP was minor (Figure S2). These findings point to FN1-GFP O-glycosylation exclusively.

\section{The L15Q polymorphism results in similar N-terminal sequences}

Although the slower form reflects distinct glycosylation, the underlying cause(s) remained to be clarified. We hypothesized that distinct glycosylation profiles might result from a shift in cleavage position of the signal peptide, as suggested by SignalP (Figure S3). Mass spectrometry of FN1-GFP fusions isolated from the culture media of NHDF however revealed 
that all forms consisted of either Gln or pyroGlu at their N-termini, consistent with previous

197 studies on full-length pFN1 ${ }^{25}$ (Figure S4). Thus, qualitative differences in N-terminal

processing are unlikely to singly account for the different glycosylation patterns. Moreover,

analysis of the gel region from the L15 sample, corresponding to a putative slower form,

identified the unequivocal presence of FN1-GFP of lower abundance ( $\sim 20 \%$ of the lower form),

\section{Quantitative differences in the secretion of the full-length FN1 variants}

The impact of the L15Q polymorphism on full-length FN1 was tested next. Due to its large size, expression of a recombinant FN1 is challenging since 1) primary cells are difficult to transfect and 2) its coding sequence is too large for lentiviral delivery. For these reasons, analyses were

207 performed on two readily transfectable cell lines: HEK293T, wherein the polymorphism had a

208 sizeable impact on the secretion of the short FN1-GFP form and HuH-7 cells, in view of the 209 major contribution of liver and wherein a significant but more modest impact on FN1-GFP

210 secretion was observed. Moreover, analysis was focused on the pFN1 given its established link 211 as a pQTL to the L15Q variant. The pFN1 construct was obtained from Addgene and modified 212 to express a C-terminal HA tag to simplify analysis. Western blot analysis revealed an

214 distinct bands in cell lysates, in contrast to the L15 which showed only one (Figure 7A). By

215 contrast, the forms recovered from the media were indistinguishable. Interestingly, the

216 additional Q15 band migrated faster than the L15 band, suggesting a lower mass, that may

217 represent an incompletely glycosylated protein. We reasoned that incomplete glycosylation 
219 mature FN1. Quantification of plasma and cellular signals indicated trends (not statistically

220 significant) in facilitated L15 secretion (increased media signal and reduced cell signal) in both

221 cell types (Figure S5). After internal correction to cellular signal however, a clear pattern

222 emerged whereby the L15 construct showed greater secretion, which approached statistical

223 significance in $\mathrm{HuH7}$ and reached it in HEK293T (Figure 7B). Thus, as for the shorter FN1-

224 GFP, the cardioprotective L15 variant seems to facilitate secretion of sFN1. 


\section{Discussion}

229

230

231

232

233

234

235

236

Here, we provide experimental evidence that the CAD protective allele of a GWAS-identified SNP increases FN1 secretion. This work provides new insights into the more global issue surrounding the role of FN1 in the pathogenesis of CAD, which is explained by genetic and environmental factors in approximately equal proportion ${ }^{26}$. Moreover, it points to the role of a common variant that contributes to the heritable component of the disease. The pervasiveness of both alleles (Global Mean Allele Frequency: 0.23/0.77) in diverse ethnic groups, albeit with an uneven geographic distribution, suggests that the CAD risk variant encoding the Q15 form may confer some evolutionary benefit.

Examination of the UK biobank data via the PheWeb interface (http://pheweb.sph.umich.edu/SAIGE-UKB/gene/FN1) demonstrates an association of FN1 with neoplasm $(\mathrm{P}=8.9 \mathrm{e}-7)$. While demonstrating that $F N 1$ may be linked to cancer, the link is with rs139452116 which results in a rare P2016L substitution and no significant association is evident for rs1250259, a SNP in strong LD with the signal peptide SNP rs1250258. This could in part account for the worsening prognosis observed previously, where strong fibronectin staining was correlated with poor survival, albeit in extreme cases only ${ }^{18}$.

Signal peptides are critical for the proper maturation and secretion of extracellular proteins. Thus, mutations within secretion signal peptides can have profound repercussions if they affect the ability of the secretory apparatus to process them. A very rare R14W mutation within the signal sequence of carbonic anhydrase IV (CA4) is linked to retinitis pigmentosa and attributed to the accumulation of the immature protein within the ER, triggering the unfolded protein response and apoptosis ${ }^{27}$. Unlike this extreme and rare example, the common L15Q substitution has modest quantitative and qualitative impacts on FN1. Impacts on glycosylation 
observed on both FN1-GFP and full-length fibronectin did not yield a coherent pattern: the Q15

252 form, while consistently less well secreted, exhibited increased glycosylation in FN1-GFP, at

253 least in some primary models, but showed reduced glycosylation of full-length FN1 in our

254 transformed cell models. Perhaps this reflects a cell-specific role of glycosylation in the control

255 of FN1 secretion or, alternatively, a subsidiary role in defining the impact of the Q15L

256 polymorphism. While typically associated with late protein maturation events, glycosylation can

257 also occur co-translationally ${ }^{28,29}$. Thus, we speculate that the L15Q variant may differentially

258 affect protein translocation through the ER channel and/or peptide cleavage kinetics, resulting in

259 altered interaction dynamics with glycosylation enzymes. For instance, slower cleavage (Q15)

within the ER lumen may increase glycosylation by facilitating transient interactions with

glycosylation enzymes. Additional investigations are required to resolve this question.

We demonstrate, using evidence from large datasets, that the cardioprotective allele is

263 linked to increased FN1 secretion suggesting that circulating FN1 protects against CAD. In

264 contrast to this hypothesis, Chiang et al employed an FN1 polymerization inhibitor to

265 demonstrate that $\mathrm{pFN} 1$ blocked vascular remodelling following carotid artery ligation in a rodent

266 model ${ }^{19}$. However, the dramatic changes entailed by carotid ligation surgery in mice combined

267 with fibronectin polymerization inhibition, which is remote from human CAD etiology. The

268 surgical intervention notwithstanding, the effect of the inhibitor on FN1 function would greatly

269 exceed any impact the common SNP may have on fibronectin expression. Thus, while a potent

270 inhibition of FN1 may protect from an early stage of atherosclerosis, a modest increase may

271 indeed be beneficial.

One limitation to our large dataset interpretation is that it is derived from an integrative

273 analysis of distinct cohorts: a UKBiobank/- and CARDIoGRAMplusC4D meta-analysis focusing 
274 on the genetics of CAD and correlative GWAS/pQTL derived from a healthy cohort ${ }^{20,24}$.

275 However, the advantage of this approach is that by examining the impact of the SNPs

276 predisposing to CAD on pFN1 levels in a largely healthy population, one avoids confounders

277 frequently associated with CAD (additional underlying conditions, medications, etc.). This

278 comes with an important limitation, as the impact of $\mathrm{pFN} 1$ levels on CAD is an extrapolation,

279 albeit an informed one.

Atherosclerosis has a complex, heterogeneous etiology, involving extensive tissue

281 remodelling characterized by smooth muscle cell proliferation which is exacerbated by

282 hypertension as well as invasion by circulating immune cells ${ }^{30-32}$. It was hypothesized that FN

283 accumulation in the aortic media may play a role in the remodelling of the aortic wall in response

284 to increased shear stress ${ }^{33}$. This is consistent with the observation that FN1 SNPs are also linked

285 to blood pressure traits, suggesting that FN1 might contribute to CAD in part through the

286 regulation of the vascular tone. Thus, the cardioprotective property of FN1 might ultimately

287 stem from its ability to regulate vascular wall ECM assembly, by jointly affecting vascular

288 elasticity and inflammation. 
Materials and Methods

\section{Tissue culture}

HuH-7 were obtained from and grown in low glucose DMEM supplemented with $1 \mathrm{~g} / \mathrm{L}$ glucose and penicillin $(0.1 \mathrm{mg} / \mathrm{ml})$ and streptomycin $(0.1 \mathrm{mg} / \mathrm{ml})$. HEK-293T and HeLa were from the ATCC and grown in DMEM with $4.5 \mathrm{~g} / \mathrm{L}$ glucose supplemented with penicillin $(0.1 \mathrm{mg} / \mathrm{ml})$ and streptomycin $(0.1 \mathrm{mg} / \mathrm{ml})$. Coronary smooth muscle cells were obtained from Sigma and the

297 ATCC and maintained in the recommended media. Human coronary adventitial fibroblasts.

298 Normal human dermal fibroblast. human aorta adventitial fibroblasts were purchased from Lonza. All primary cells were maintained in their recommended media.

\section{DNA constructs}

301 The short GFP-HA fusion proteins (L15 and Q15) were obtained by chemical synthesis of two dsDNA block variants (BioBasic) encoding amino acid 1-182 of FN1 and inserted via restriction

303 cloning in pLVX-puro digested with EcoRI/BamHI. The construct, referred to as FN1-GFP

304 throughout, included C-terminal GFP and HA tags. The full-length pFN1 construct was obtained

305 from Addgene (Fibronectin-human-plasma in pMAX; Plasmid \#120401 ${ }^{34}$ ). A Q15L

306 substitution was achieved by Q5 mutagenesis (New England Biolab) on a N-terminal Hind III/AvrII fragment transferred in pCMV5 digested similarly. Following validation by Sanger

308 sequencing, the fragment was returned to the pMAX construct. A Hemagglutinin A epitope tag 


\section{Transfection and transduction}

314 Cells were transfected with lipofectamine 3000 (ThermoFisher) using a ratio of 3:2:1

315 (lipofectamine $3000(\mu \mathrm{l})$ : P3000 reagent $(\mu \mathrm{l})$ : DNA $(\mu \mathrm{g})$ ). For infection, viral particles were first 316 generated in HEK-293FT cells using PVLX-puro (Clontech) alongside psPAX2 and pMD2.G

317 obtained from Addgene. Virus containing supernatants were filtered through $400 \mathrm{~nm}$ filters and 318 frozen at $-80 \mathrm{C}$ as is. Infections were performed in the presence of polybrene $(2 \mu \mathrm{g} / \mathrm{ml})$.

\section{Secretion assays}

For secretion assays, the short (FN1-GFP) construct, cells stably transduced with a pLVX FN1-

321 GFP were grown in phenol-free media for 48-72 $\mathrm{h}$ prior to assay. Media was recovered while

322 cells were rinsed in PBS and lysed in PBS/1\% Triton X-100. Aliquots of cell lysates and media

323 were then transferred to assay plates and GFP fluorescence was quantified by fluorometry on a

324 fluorescence microplate reader (Ex 470, Em 510; BioTek). After subtraction of background

325 values, fluorescence from media was divided by cell fluorescence. To measure FN1-GFP

326 synthesis within HEK293T (Figure 2), FN1-GFP together with pRSV40 Renilla (2\% of total

327 transfected DNA) were transiently transfected and lysed in Passive Lysis Buffer (Promega). Cell

328 lysates were analyzed by Western blot while Renilla luciferase activity (Glomax luminometer,

329 Promega) was used to correct for transfection efficiency.

330 For the full-length FN1-HA constructs, cells (in 24 well plate format) were transiently

331 transfected for $24 \mathrm{~h}$ with $0.2 \mathrm{ug}$ of pMAX-FN1 and $4 \mathrm{ng}$ of pRSV40 Renilla. Media were

332 recovered while cells were rinsed in PBS and lysed in Passive Lysis Buffer (Promega). Media

333 was directly subjected to Western Blotting, while lysates were used to normalize the expression

334 values by measuring Renilla luciferase activity 


\section{Immunoprecipitation and Western blotting}

337 Cells were lysed in IP buffer (50 mM Tris-HCl, pH 7.4, 0.15 M NaCl, 0.1\% Nonidet P40

(IGEPAL), $5 \mathrm{mM} \mathrm{MgCl} 2$ ) for $2 \mathrm{~min}$ at $4{ }^{\circ} \mathrm{C}$. Lysates ( $1 \mathrm{mg}$ protein equivalent) were then cleared

by centrifugation $(17,000 \mathrm{Xg})$ for $5 \mathrm{~min}$ and $20 \mu \mathrm{l}$ of prewashed Anti-HA magnetic beads

(Pierce) were added. For isolation from the media, $20 \mu 1$ of beads were added to $3 \mathrm{ml}$ of $400 \mathrm{~nm}$

341 filtered media harvested $72 \mathrm{~h}$ post-infection. Western blot was performed using 8 or $10 \%$ mini

342 gels followed by wet transfer $(1 \mathrm{~h}, 100 \mathrm{~V})$ to Western grade nitrocellulose (Bio-Rad). Blots were

343 incubated in Intercept blocking buffer (LI-COR) for $1 \mathrm{~h}$ and incubated for $16 \mathrm{~h}$ at $4{ }^{\circ} \mathrm{C}$ in the

344 presence of cognate primary antibodies diluted 1:2000 in TBS/T (50 mM Tris-HCl, pH 7.4, 0.15

$345 \mathrm{M} \mathrm{NaCl}, 0.1 \%$ Tween-20). Secondary antibodies (donkey anti-mouse (680) or -rabbit (700); LI-

346 COR) were diluted 1:20,000. Four 1 min washes in PBS were performed after each antibody

347 incubation.

\section{RNA isolation and qRT-PCR}

349 RNA was isolated using the High Pure Isolation Kit (Roche). The Transcriptor First Strand 350 cDNA Synthesis Kit (Roche) was used to generate cDNA using a 1:1 mixture of random

351 hexamer and oligodT. PCR amplification and quantification were performed on a Roche

352 LightCycler 480 using the SYBR Green I Master reaction mix (Roche). For each experiment,

353 relative amounts of target cDNAs were first expressed relative to SRP14. Results shown

354 represent the means of 3 biological replicates. Oligonucleotides used are described in

355 Supplemental Materials. 


\section{Mendelian Randomization}

To investigate the possibility of an association between plasma protein level of FN1 and CAD, we did multi-SNP summary-based Mendelian randomization (MR) analysis which is also known as 2-sample Mendelian randomization ${ }^{35}$. For this purpose, we obtained summary association statistics (Beta and Standard error) for SNPs (pQTLs) that are independently $\left(\mathrm{r}^{2}<0.2\right)$ associated $\left(\mathrm{P}<5 \mathrm{e}^{-8}\right)$ with $\mathrm{FN} 1$ protein level and used these as an instrument to investigate a causal effect. This means, for SNPs in our instrument $\left(\mathrm{MR} \mathrm{N}_{\mathrm{SNP}}\right)$, we also obtained their summary association statistics (Beta and Standard error) with CAD and contrasted the effect sizes of the SNPs on FN1 (exposure) with the effect sizes of the SNPs on the CAD (outcome), to estimate the causal effect of FN1 on CAD. In this context, a significant negative association indicates individuals genetically susceptible to have higher levels of FN1 are at lower risk of CAD. MR analysis was done using the GSMR (Generalised Summary-data-based Mendelian Randomisation) algorithm implemented in GCTA software (version 1.92) ${ }^{35}$. As compared to other methods for 2-sample MR analysis, GSMR automatically detects and removes SNPs that have pleiotropic effect on both exposure and outcome using the HEIDI test; in addition, GSMR accounts for the sampling variance in $\beta$ (beta) estimates and the linkage disequilibrium (LD) among SNPs, as such it is statistically more powerful than other 2-sample MR approaches. GSMR also incorporates a variety of quality assurance and helpful functions, notably aligning both GWAS summary datasets to the same reference allele at each SNP. Excluding SNPs that difference between their allele frequency in GWAS summary datasets and the LD reference sample is greater than 0.2 , a clumping function to only keep non-correlated $\left(\mathrm{r}^{2}<0.2\right)$ SNPs (with association P-value $\left.<5 \mathrm{e}^{-8}\right)$ in the instrument and a function to generate the scatter plot of SNP effects. Previously we used this approach to investigate the role of circulating miRNAs with regard to cardiometabolic 
380

381

382

383

384

385

386

387

388

389

390

391

392

393

394

395

396

397

398

399

400

401

402

phenotypes ${ }^{36}$. We obtained GWAS summary statistics for CAD from the most recent metaanalysis of CARDIoGRAMplusC4D and UK Biobank ${ }^{20}$ and GWAS summary statistics for SNPs that influence FN1 protein level from Suhre et al ${ }^{24}$.

\section{Deglycosylation reactions}

Culture media from Q15 and L15 NHDF infected for $96 \mathrm{~h}$ with lentiviral constructs expressing FN1-GFP were recovered, supplemented with $1 \mathrm{mM}$ PMSF and centrifuged (1000 X g, 2 min) to remove cellular debris, and further cleared at high speed for $5 \min (13,000 \mathrm{X} \mathrm{g})$. Recombinant FN1-GFP was isolated from $10 \mathrm{ml}$ of media (corresponding to a $10 \mathrm{~cm}$ culture dish) using $25 \mu \mathrm{l}$ anti-HA Pure Proteome magnetic beads (Pierce). Beads were washed 4 X $0.5 \mathrm{ml}$ of PBS/1 \% Triton X-100 and resuspended in $250 \mu 1$ of the same buffer. Aliquots (10\%) of the isolates were used per deglycosylation reaction. Deglycosylation was performed using the Protein Deglycosylation Mix II according to the supplier's protocol (New England Biolab); the kit includes a mixture of PNGase F, O-glycosydase and exoglycosydases to remove most glycans from target proteins. Briefly, the immunoisolated material was denatured for $10 \mathrm{~min}$ at $75^{\circ} \mathrm{C}$ and subjected to a deglycosylation reaction for $30 \mathrm{~min}$ at $20^{\circ} \mathrm{C}$ and $180 \mathrm{~min}$ at $37 \mathrm{C}$, using enzyme mix $(2.5 \mu 1)$ or a mock reaction (no enzyme mix) in $25 \mu 1$ of bead suspension. Samples were then denatured in reducing SDS-PAGE sample buffer and analyzed by Western blotting.

\section{Protein Analysis by LC-MS/MS}

For mass spectrometry, Q15 and L15 FN1-GFP samples were immunoprecipitated from the media of transduced NHDF as described above, resolved by reducing SDS-PAGE and stained by colloidal Coomassie blue (Simply blue); NHDF were chosen for their greater proliferative ability over coronary models while exhibiting similar shifts on SDS-PAGE. Gel pieces were than excised and destained; a gel area matching a putative, lower abundance glycosylated L form was 
403 also included, for a total of 4 samples. Two distinct biologics per sample were analyzed.

404 Proteomics analysis was performed at the Ottawa Hospital Research Institute Proteomics Core

405 Facility (Ottawa, Canada). Proteins were digested in-gel using trypsin (Promega) according to

406 the method of Shevchenko ${ }^{37}$. Peptide extracts were concentrated by Vacufuge (Eppendorf).

407 LC-MS/MS was performed using a Dionex Ultimate 3000 RLSC nano HPLC (Thermo

408 Scientific) and Orbitrap Fusion Lumos mass spectrometer (Thermo Scientific). MASCOT

409 software version 2.6.2 (Matrix Science, UK) was used to infer peptide and protein identities from

410 the mass spectra. The observed spectra were matched against custom sequences and against an

411 in-house database of common contaminants. The results were exported to Scaffold (Proteome

412 Software, USA) for further validation and viewing.

\section{Statistical analysis}

414 To estimate statistical significance of experimental findings, unpaired Student t-test (2-tailed)

415 were performed in GraphPad Prism. 
424

425

426

427

428

429

430

431

432

433

434

435

436

437

438

439

440

441

442

443

444

\section{References}

1. van der Harst, P. \& Verweij, N. Identification of 64 Novel Genetic Loci Provides an Expanded View on the Genetic Architecture of Coronary Artery Disease. Circ. Res. 122, 433-443 (2018).

2. Warren, H. R. et al. Genome-wide association analysis identifies novel blood pressure loci and offers biological insights into cardiovascular risk. Nat. Genet. 49, 403-415 (2017).

3. Nelson, C. P. et al. Association analyses based on false discovery rate implicate new loci for coronary artery disease. Nat. Genet. 49, 1385-1391 (2017).

4. Nicolae, D. L. et al. Trait-Associated SNPs Are More Likely to Be eQTLs: Annotation to Enhance Discovery from GWAS. PLoS Genet. 6, e1000888 (2010).

5. Nica, A. C. et al. Candidate causal regulatory effects by integration of expression QTLs with complex trait genetic associations. PLoS Genet. 6, e1000895 (2010).

6. Schaid, D. J., Chen, W. \& Larson, N. B. From genome-wide associations to candidate causal variants by statistical fine-mapping. Nat. Rev. Genet. 19, 491-504 (2018).

7. Wu, Y., Zheng, Z., Visscher, P. M. \& Yang, J. Quantifying the mapping precision of genome-wide association studies using whole-genome sequencing data. Genome Biol. 18, 86 (2017).

8. Liu, X., Li, Y. I. \& Pritchard, J. K. Trans Effects on Gene Expression Can Drive Omnigenic Inheritance. Cell 177, 1022-1034.e6 (2019).

9. Cannon, M. E. \& Mohlke, K. L. Deciphering the Emerging Complexities of Molecular Mechanisms at GWAS Loci. Am. J. Hum. Genet. 103, 637-653 (2018). 
445 10. Gupta, R. M. et al. A Genetic Variant Associated with Five Vascular Diseases Is a Distal Regulator of Endothelin-1 Gene Expression. Cell 170, 522-533.e15 (2017).

447 11. Smemo, S. et al. Obesity-associated variants within FTO form long-range functional connections with IRX3. Nature 507, 371-375 (2014).

12. Wang, Y. et al. Comprehensive Cis-Regulation Analysis of Genetic Variants in Human Lymphoblastoid Cell Lines. Front. Genet. 10, 806 (2019).

451 13. To, W. S. \& Midwood, K. S. Plasma and cellular fibronectin: Distinct and independent functions during tissue repair. Fibrogenesis and Tissue Repair 4, 1-17 (2011).

14. Gutman, A., Yamada, K. M. \& Kornblihtt, A. Human fibronectin is synthesized as a prepropolypeptide. FEBS Lett. 207, 145-8 (1986).

15. Sakai, T. et al. Plasma fibronectin supports neuronal survival and reduces brain injury following transient focal cerebral ischemia but is not essential for skin-wound healing and

16. Moretti, F. A. et al. A major fraction of fibronectin present in the extracellular matrix of tissues is plasma-derived. J. Biol. Chem. 282, 28057-28062 (2007).

17. Kumra, H. et al. Roles of fibronectin isoforms in neonatal vascular development and matrix integrity. PLoS Biol. 16, e2004812 (2018).

462 18. von Au, A. et al. Circulating fibronectin controls tumor growth. Neoplasia 15, 925-938 (2013).

464 19. Chiang, H. Y., Korshunov, V. A., Serour, A., Shi, F. \& Sottile, J. Fibronectin is an important regulator of flow-induced vascular remodeling. Arterioscler. Thromb. Vasc. 
Biol. 29, 1074-1079 (2009).

467 20. van der Harst, P. \& Verweij, N. Identification of 64 Novel Genetic Loci Provides an Expanded View on the Genetic Architecture of Coronary Artery Disease Novelty. Circ. Res. 122, 433-443 (2018).

470

21. Staley, J. R. et al. PhenoScanner: A database of human genotype-phenotype associations.

471 Bioinformatics 32, 3207-3209 (2016).

22. Kamat, M. A. et al. PhenoScanner V2: An expanded tool for searching human genotypephenotype associations. Bioinformatics 35, 4851-4853 (2019).

474 23. Emilsson, V. et al. Co-regulatory networks of human serum proteins link genetics to disease. Science. 361, 769-773 (2018).

24. Suhre, K. et al. Connecting genetic risk to disease end points through the human blood plasma proteome. Nat. Commun. 8, 14357 (2017).

25. Garcia-Pardo, A., Pearlstein, E. \& Frangione, B. Primary structure of human plasma fibronectin. J. Biol. Chem. 260, 10320-10325 (1985).

26. McPherson, R. \& Tybjaerg-Hansen, A. Genetics of Coronary Artery Disease. Circ. Res. 118, 564-578 (2016).

27. Rebello, G. et al. Apoptosis-inducing signal sequence mutation in carbonic anhydrase IV identified in patients with the RP17 form of retinitis pigmentosa. Proc. Natl. Acad. Sci. U. S. A. 101, 6617-6622 (2004). binding during glycoprotein synthesis. Proc. Natl. Acad. Sci. U. S. A. 92, 6229-6233 
(1995).

488

29. Ruiz-Canada, C., Kelleher, D. J. \& Gilmore, R. Cotranslational and Posttranslational NGlycosylation of Polypeptides by Distinct Mammalian OST Isoforms. Cell 136, 272-283 (2009).

30. Laurent, S. \& Boutouyrie, P. The Structural Factor of Hypertension: Large and Small Artery Alterations. Circulation Research 116, 1007-1021 (2015).

31. Brown, I. A. M. et al. Vascular smooth muscle remodeling in conductive and resistance arteries in hypertension. Arterioscler. Thromb. Vasc. Biol. 38, 1969-1985 (2018).

32. Intengan, H. D. \& Schiffrin, E. L. Vascular remodeling in hypertension: roles of apoptosis, inflammation, and fibrosis. Hypertension 38, 581-587 (2001).

33. Bézie, Y. et al. Fibronectin expression and aortic wall elastic modulus in spontaneously hypertensive rats. Arterioscler. Thromb. Vasc. Biol. 18, 1027-1034 (1998).

34. Rossnag1, S. et al. EDA-Fibronectin Originating from Osteoblasts Inhibits the Immune Response against Cancer. PLoS Biol. 14, e1002562 (2016).

35. Zhu, Z. et al. Causal associations between risk factors and common diseases inferred from GWAS summary data. Nat. Commun. 9, 224 (2018).

36. Nikpay, M. et al. Genome-wide identification of circulating-miRNA expression quantitative trait loci reveals the role of several miRNAs in the regulation of cardiometabolic phenotypes. Cardiovasc. Res. 115, 1629-1645 (2019).

37. Shevchenko, A., Tomas, H., Havliš, J., Olsen, J. V. \& Mann, M. In-gel digestion for mass spectrometric characterization of proteins and proteomes. Nat. Protoc. 1, 2856-2860 
bioRxiv preprint doi: https://doi.org/10.1101/2021.03.09.434522; this version posted April 5, 2021. The copyright holder for this preprint (which was not certified by peer review) is the author/funder, who has granted bioRxiv a license to display the preprint in perpetuity. It is made available under aCC-BY 4.0 International license. 


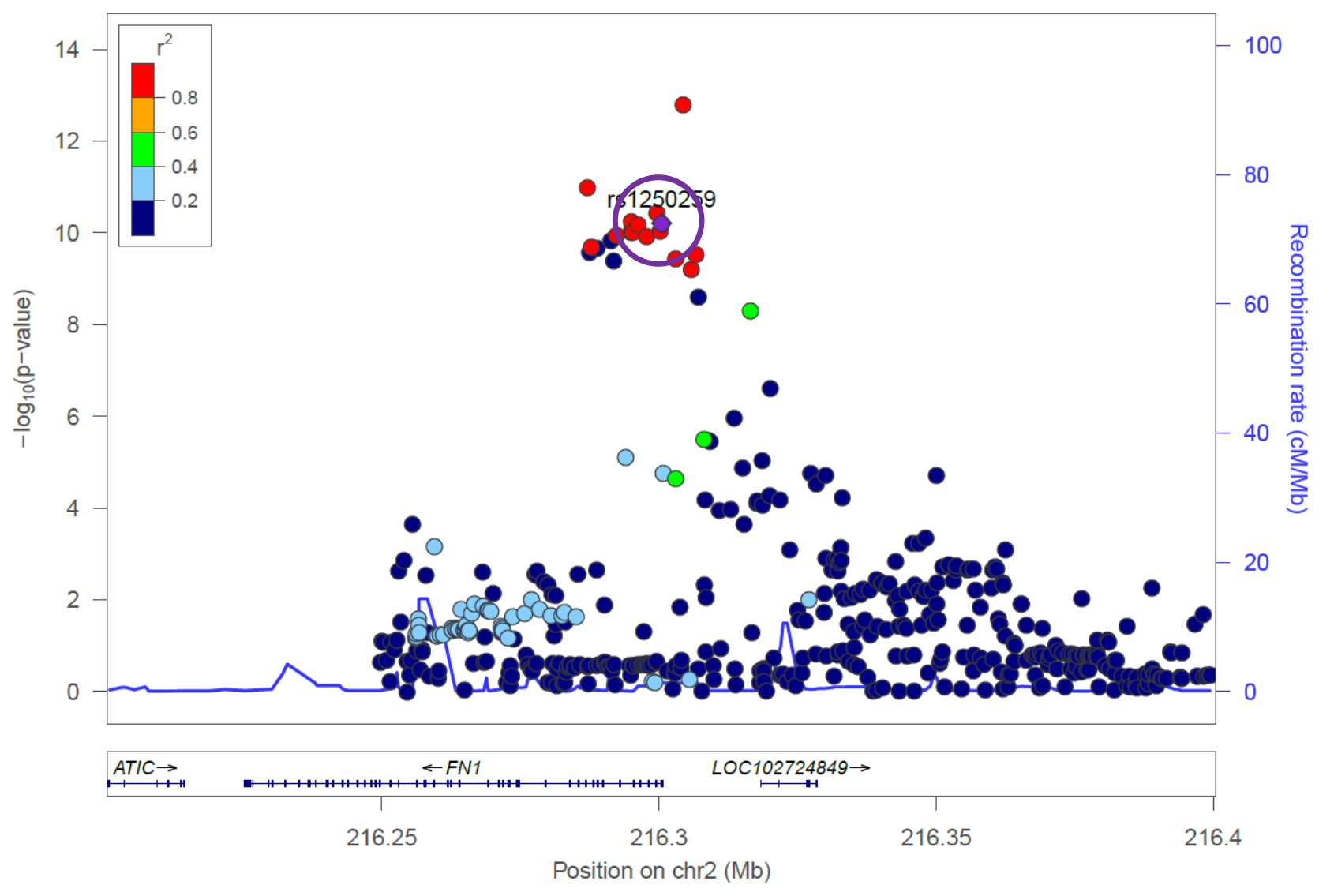

Figure 1. Local Manhattan plot of CAD association. CAD association data centred on rs1250259 ( $\pm 0.2 \mathrm{Mb})$, in purple and circled, from Van der Harst (https://doi.org/10.1161/CIRCRESAHA.117.312086) plotted using LocusZoom, showing a signal enrichment around the upstream region of FN1. 

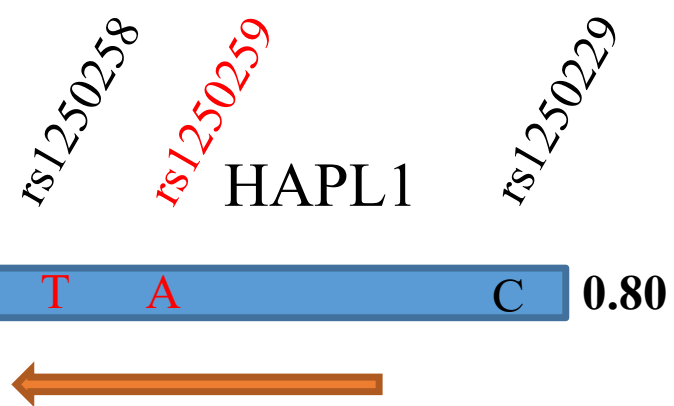

FN1 L15

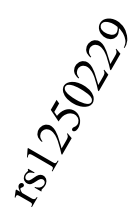

0.80

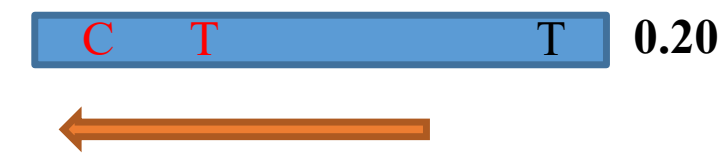

FN1 Q15

Figure 2. Haplotype structure around rs1250229, linked to both CAD and blood pressure. The C allele at rs1250229 correlates with the presence of $\mathrm{rs} 1250259-\mathrm{A}\left(\mathrm{r}^{2}=0.94\right)$, resulting in $\mathrm{T}$ on the coding strand of FN1 which is expressed from the negative strand. The corresponding codon (CTG) encodes a Leucine at position 15 of FN1 while the alternate codon (CAG) codes for Glutamine. Numbers on the right (Bold) are the fraction of the corresponding phased haplotype over the total number of observed haplotypes. Values are from the 1000 Genomes Project, using rs1250259 values (all populations). Genotype information for rs 125058 and 59 were verified and found to be consistent with the Ottawa cohort genotyping. 
A

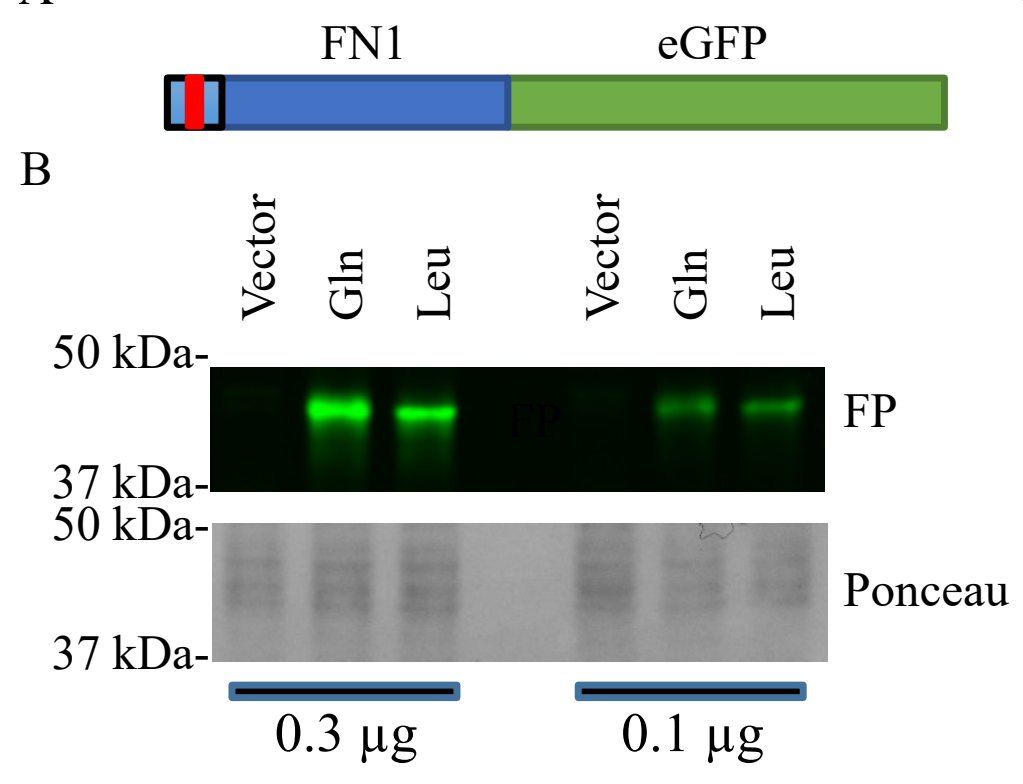

$\mathrm{C}$

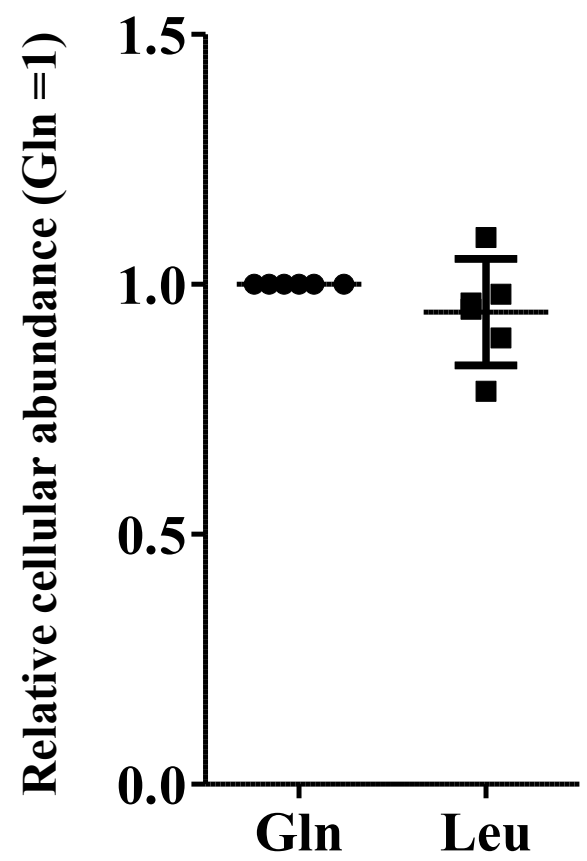

Figure 3. Both FN1 variants express similarly in HEK293T cells. A, Schema of the FN1-FP construct used. Drawing is approximately to scale. FN1 region corresponds to AA1-182, which encompasses, the signal peptide as well as 3 complete Fibronectin type-I domains corresponding to a previously reported crystal structure (2CG7, PDB entry). Signal peptide is in lighter blue. Red bar indicates position of the L15Q polymorphism. B,C, Analyses of cell lysates transfected with either variant (or vector alone). In B, HEK293T cells were transfected for $48 \mathrm{~h}$ with constructs encoding FN1-GFP fusion proteins, lysed and resolved by SDS-PAGE. The Gln variant exhibited a slight retardation relative to the Leu variant. In C, quantification of the cellular FP intensity in lysates after correction for transfection efficiency (Renilla). Each data point represents an independent experimental replicate. 


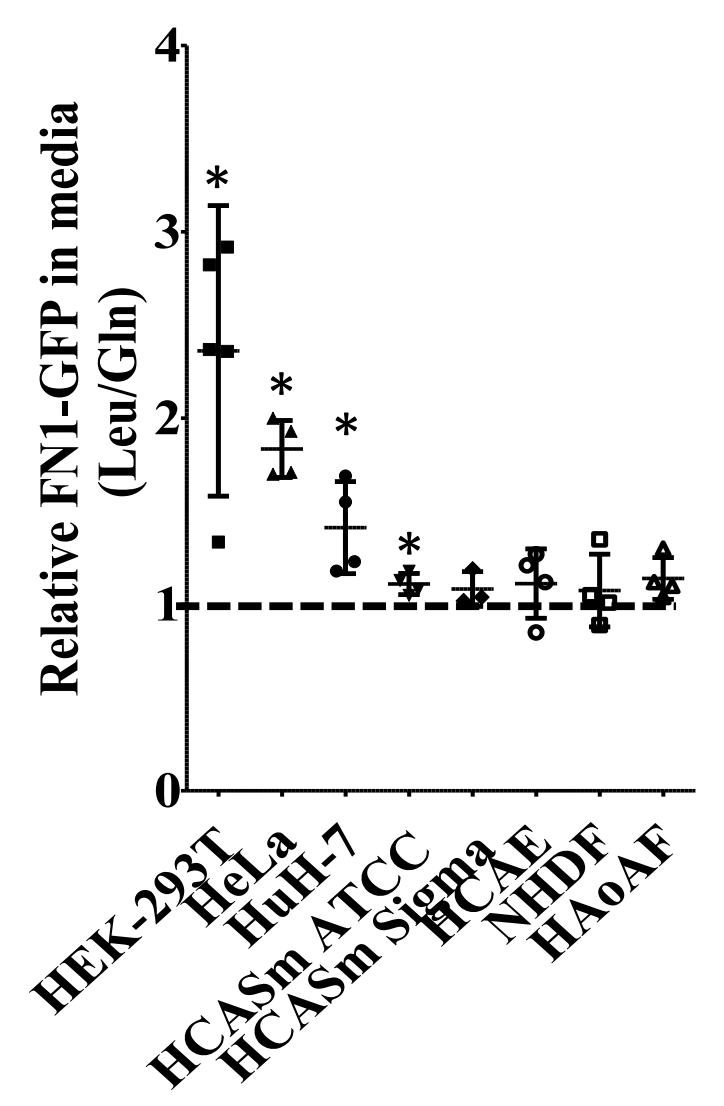

Figure 4. Presence of the CAD protective allele results in increased secretion of a FN1 model

construct. Media and lysates from cells transduced for 48 hours with FN1-GFP plasmids encoding either Leu 15 or Gln 15 were analyzed by fluorometry. After correction for background signal, ratios of media to cellular GFP fluorescence were first assessed for each variant (L/Q) and the values for the Leu allele were divided by the corresponding Gln values. Values above 1 represent an enrichment of the L15 form.

Results represent the mean from 3-5 independent determinations $\pm 95 \%$ C.I. HCASm: human coronary artery smooth muscle cells from either ATCC or Sigma; HCAE: human coronary artery endothelial cells; NHDF: Normal Human Dermal fibroblast; HAoAF: Human aorta Adventitial fibroblast. 


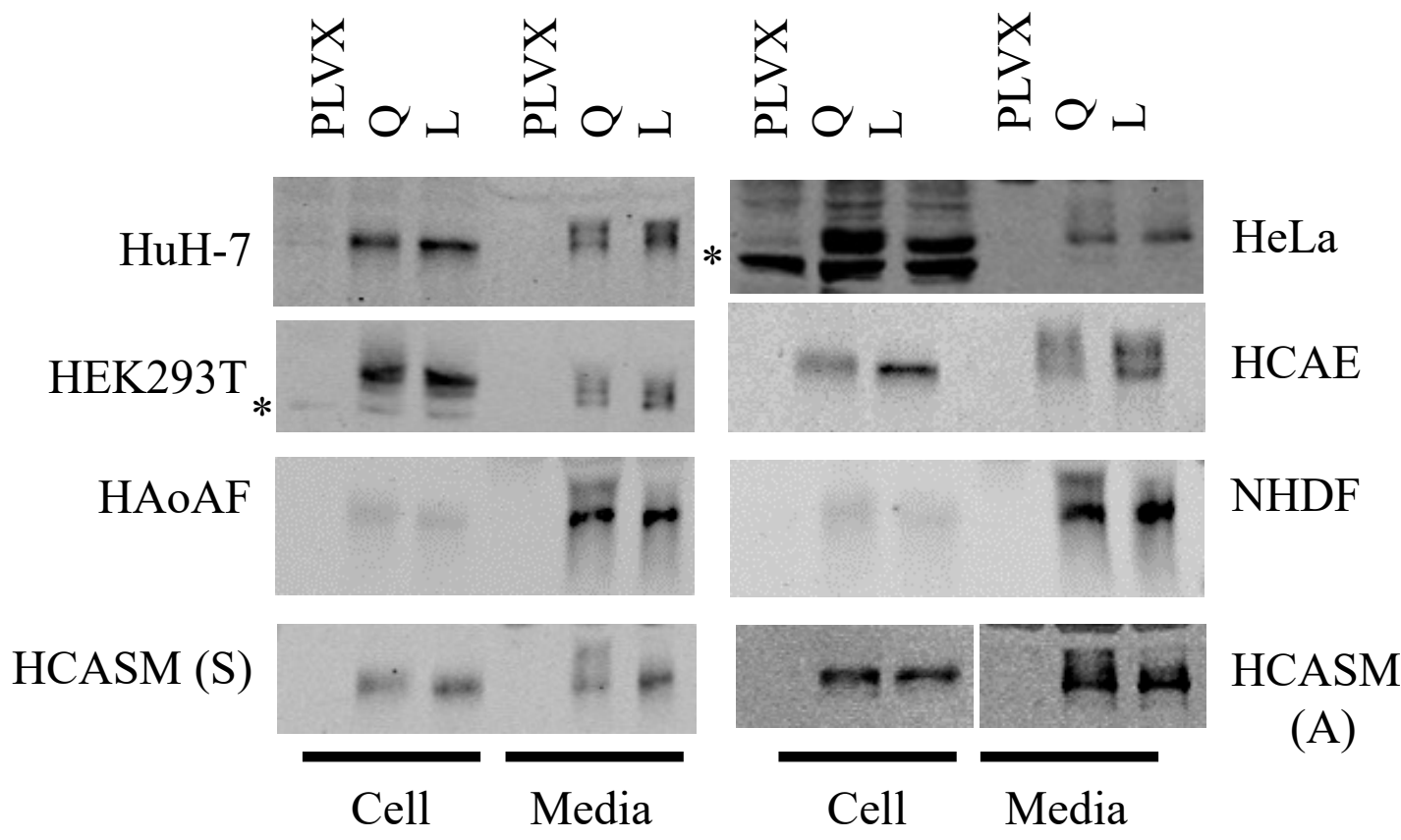

Figure 5. Multiple FN1-GFP species are secreted in cell cultures. Media ( $2 \%$ total well) and lysates (10\% total well) from cells transduced for 72 hours with FN1-GFP plasmids encoding either L15 (T allele) or Q15 (A allele) were analyzed by Western blot using GFP antibodies (Sigma for all except for HeLa, Invitrogen). HCAE: human coronary artery endothelial cells; NHDF: Normal Human Dermal Fibroblast; HAoAF: Human aorta Adventitial fibroblast; HCASM: human coronary artery smooth muscle cells from either ATCC (A) or Sigma (S). Data is representative of at least 3 independent experiments. * indicates a non-specific band. Regions shown span the 37 to $50 \mathrm{kDa}$ range. 


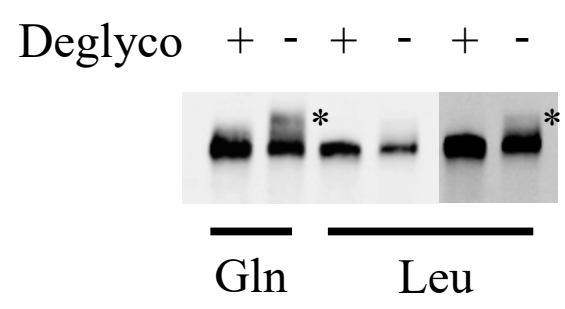

Figure 6. Glycosylation patterns of Q15 and L15 FN1-GFP from dermal fibroblast differ. Media from NHDF transduced for 72 hours with FN1-GFP plasmids encoding either L15 (T allele) or Q15 (A allele) were recovered by immunoprecipitation with anti-HA beads, denatured and treated with (+) or without (-) deglycosylation enzymes prior to Western blotting using an anti-GFP antibody. A higher exposure of the Leu samples is included to facilitate L-Q comparison. * indicates the position of a larger, glycosylated form. Regions shown span the 37 to $50 \mathrm{kDa}$ range 
A

\section{Cell Media Cell Media}

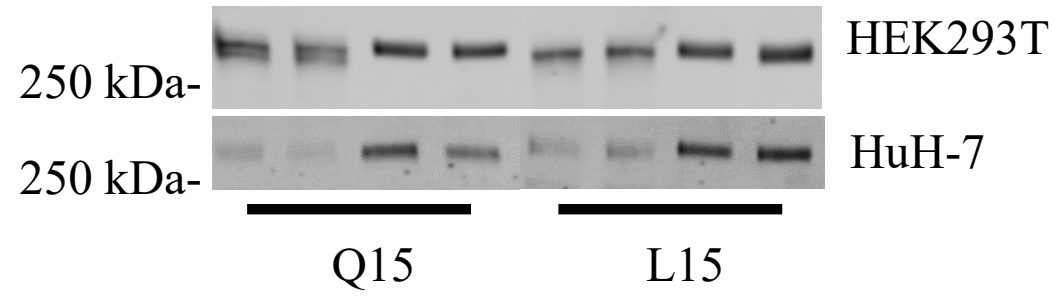

B

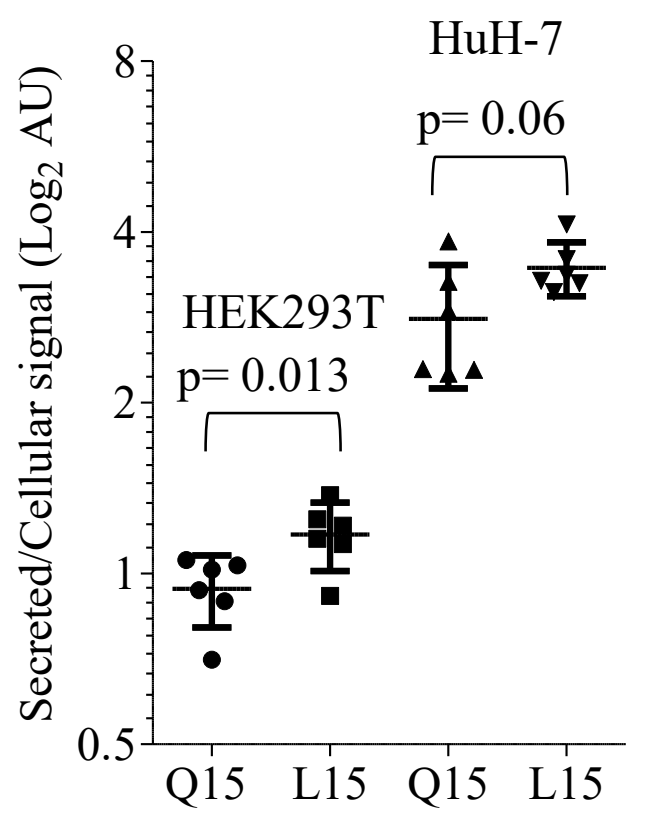

Figure 7. The full-length FN1 Q15 variant is less efficiently processed and secreted. Constructs encoding either variant of pFN1, tagged with a Cterminal Hemagglutinin tag, were transfected for 22 $h$ in HEK293T or HuH-7 cells, as indicated A, Media and lysates were then analyzed by Western blot using an HA-specific antibody; no signal was detected in untransfected cells. Two independent experiments are shown for each construct. B, Quantification of FN1-HA Western blots. Signals from media (GFP) and cytosol (pRSV40 Renilla) were quantified for each transfection and data is expressed as the secreted to cellular signals for Q15 and L15 ( $\pm 95 \%$ C.I.). Each point represents a distinct experiment. 